\title{
SHOCK EXCITED MOLECULES IN NGC 1266: ULIRG CONDITIONS AT THE CENTER OF A BULGE-DOMINATED GALAXY
}

\author{
E. W. Pellegrini ${ }^{1}$, J. D. Smith (PI) ${ }^{1}$, M. G. Wolfire $^{2}$, B. T. Draine ${ }^{3}$, A. F. Crocker ${ }^{1}$, K. V. Croxall ${ }^{4}$, P. van der Werf $^{5}$, \\ D. A. Dale ${ }^{6}$, D. Rigopoulou ${ }^{7,8}$, C. D. Wilson ${ }^{9}$, E. Schinnerer ${ }^{10}$, B. A. Groves ${ }^{10}$, K. Kreckel ${ }^{10}$, K. M. Sandstrom $^{10}$, \\ L. Armus $^{11}$, D. Calzetti ${ }^{12}$, E. J. Murphy ${ }^{11}$, F. WAlter ${ }^{10}$, J. Koda ${ }^{13}$, E. Bayet ${ }^{14}$, P. Beirao ${ }^{15}$, A. D. Bolatto $^{2}$,

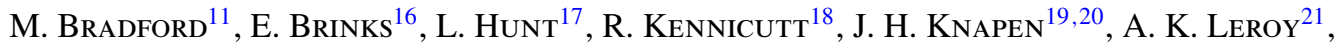 \\ E. RosolowsKY ${ }^{22}$, L. Vigroux ${ }^{23}$, AND R. H. B. HopwOOD ${ }^{24}$ \\ ${ }^{1}$ Department of Physics and Astronomy, University of Toledo, Toledo, OH 43606, USA; eric.pellegrini@ @utoledo.edu \\ 2 Department of Astronomy, University of Maryland, College Park, MD 20742, USA \\ ${ }^{3}$ Princeton University Observatory, Peyton Hall, Princeton, NJ 08544-1001, USA \\ ${ }^{4}$ Department of Astronomy, The Ohio State University, 140 West 18 th Avenue, Columbus, OH 43210, USA \\ ${ }^{5}$ Leiden Observatory, Leiden University, P.O. Box 9513, 2300-RA Leiden, The Netherlands \\ ${ }^{6}$ Department of Physics and Astronomy, University of Wyoming, Laramie, WY 82071, USA \\ ${ }^{7}$ Department of Astrophysics, University of Oxford, Keble Road, Oxford OX1 3RH, UK \\ ${ }^{8}$ RAL Space, Rutherford Appleton Laboratory, Chilton, Didcot OX110QX, UK \\ ${ }^{9}$ Department of Physics and Astronomy, McMaster University, Hamilton, Ontario L8S 4M1, Canada \\ ${ }^{10}$ Max Planck Institut fur Astronomie, Konigstuhl 17, D-69117 Heidelberg, Germany \\ ${ }^{11}$ Spitzer Science Center, California Institute of Technology, MC 314-6, Pasadena, CA 91125, USA \\ 12 Department of Astronomy, University of Massachusetts, Amherst, MA 01003, USA \\ ${ }^{13}$ Department of Physics and Astronomy, SUNY Stony Brook, Stony Brook, NY 11794-3800, USA \\ ${ }^{14}$ Department of Physics and Astronomy, University College London, Gower Street, London WC1E 6BT, UK \\ ${ }^{15}$ Observatoire de Paris, 61 avenue de l'Observatoire, Paris F-75014, France \\ ${ }^{16}$ Centre for Astrophysics Research, University of Hertfordshire, Hatfield AL 10 9AB, UK \\ 17 INAF-Osservatorio Astrosico di Arcetri, Largo E. Fermi 5, I-50125 Firenze, Italy \\ ${ }^{18}$ Institute of Astronomy, University of Cambridge, Madingley Road, Cambridge CB3 0HA, UK \\ ${ }^{19}$ Instituto de Astrofsica de Canarias, E-38200 La Laguna, Spain \\ ${ }^{20}$ Departamento de Astrofísica, Universidad de La Laguna, E-38206 La Laguna, Spain \\ ${ }^{21}$ National Radio Astronomy Observatory, 520 Edgemont Road, Charlottesville, VA 22903, USA \\ 22 Department of Physics, University of Alberta, 2-115 Centennial Centre for Interdisc Science, Edmonton AB, Canada \\ ${ }^{23}$ Institut dAstrophysique de Paris, UMR 7095 CNRS, Universite Pierre et Marie Curie, F-75014 Paris, France \\ ${ }^{24}$ Department of Physics and Astronomy, Open University, Walton Hall, Milton Keynes MK7 6AA, UK \\ Received 2013 August 29; accepted 2013 November 15; published 2013 December 2
}

\begin{abstract}
We investigate the far infrared (IR) spectrum of NGC 1266, a S0 galaxy that contains a massive reservoir of highly excited molecular gas. Using the Herschel Fourier Transform Spectrometer, we detect the ${ }^{12} \mathrm{CO}$ ladder up to $J=(13-12),\left[\mathrm{CI}_{\mathrm{I}}\right]$ and $[\mathrm{N} \mathrm{II}]$ lines, and also strong water lines more characteristic of UltraLuminous IR Galaxies (ULIRGs). The ${ }^{12} \mathrm{CO}$ line emission is modeled with a combination of a low-velocity $\mathrm{C}$-shock and a photodissociation region. Shocks are required to produce the $\mathrm{H}_{2} \mathrm{O}$ and most of the high- $J$ CO emission. Despite having an IR luminosity 30 times less than a typical ULIRG, the spectral characteristics and physical conditions of the interstellar medium of NGC 1266 closely resemble those of ULIRGs, which often harbor strong shocks and large-scale outflows.
\end{abstract}

Key words: galaxies: individual (NGC 1266) - infrared: ISM - ISM: jets and outflows - ISM: lines and bands ISM: molecules - photon-dominated region (PDR)

Online-only material: color figures

\section{INTRODUCTION}

The IR emission of galaxies beyond the peak of their thermal dust emission, from 200-600 $\mu \mathrm{m}$, traces their ionized, neutral, and molecular interstellar medium (ISM). In particular the ${ }^{12} \mathrm{CO}$ transitions (hereafter $\mathrm{CO}$ ) from $J=4-3$ to $J=13-12$ provide an important diagnostic of molecular gas excitation. These CO spectral line energy distributions (SLEDs) allow one to distinguish those regions within galaxies dominated by star formation (SF; photodissociation regions (PDRs, also referred to as photon-dominated regions)) or by active galactic nucleus (AGN) produced X-ray dominated regions (XDRs).

At a distance of $29.9 \mathrm{Mpc}(z=0.0073)$, NGC 1266 contains $1.1 \times 10^{9} M_{\odot}$ of molecular gas within a $100 \mathrm{pc}$ radius, with a denser, $60 \mathrm{pc}$ core containing $4 \times 10^{8} M_{\odot}$ of gas (Alatalo et al. 2011). In contrast, nearby giant molecular clouds of a similar size contain only $10^{6} M_{\odot}$ of gas
(Fukui \& Kawamura 2010). The core mass and projected size corresponds to a surface density of $2.7 \times 10^{4} M_{\odot} \mathrm{pc}^{-2}$, similar to what is observed in UltraLuminous IR Galaxies (ULIRGs; Bothwell et al. 2010). Its IRAS 60/100 $\mu \mathrm{m}$ ratio is twice as large as the median value of more ordinary galaxies within the SINGS sample (Dale et al. 2009), yet within 5\% of the mean ratio of 41 ULIRGs (Farrah et al. 2003). The observed $9.7 \mu \mathrm{m}$ silicate optical depth $\tau(\mathrm{Si}) \approx 2$ (Spitzer-Infrared Spectrograph spectra; Smith et al. 2007). The ISM column density and dust colors are quite comparable with samples of ULIRGS, despite NGC 1266's total infrared luminosity (TIR) of only $2.75 \times 10^{10} L_{\odot}$.

Broad velocity wings of the low- $J \mathrm{CO}$ lines reveal a massive and energetic molecular outflow $\left(M_{\text {out }}=2.4 \times 10^{7} M_{\odot}, \dot{M}_{\text {out }}=\right.$ $13 M_{\odot} \mathrm{yr}^{-1}$; Alatalo et al. 2011). The mass outflow rate exceeds the SF rate by a factor of five, leading to a highly obscured, weak AGN as a likely driving mechanism (Alatalo et al. 2011). 


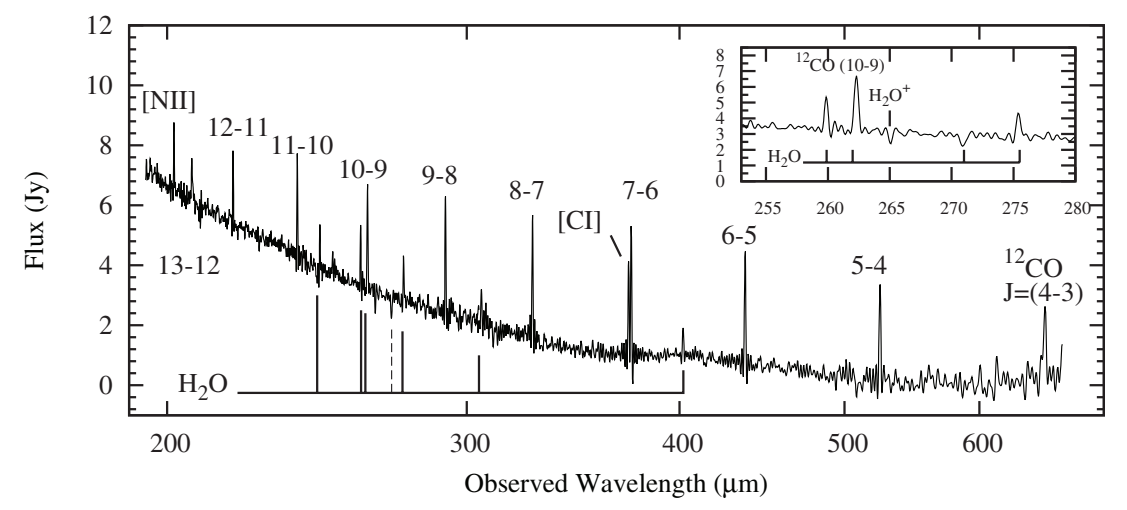

Figure 1. SPIRE-FTS spectrum from 200 to $600 \mu \mathrm{m}$. Emission lines include ${ }^{12} \mathrm{CO}$ from $J=(4-3)$ to $J=(13-12)$, [N II] 205, [C I] 370 and $609 \mu \mathrm{m}$. Strong ortho and para $\mathrm{H}_{2} \mathrm{O}$ emission characteristic of ULIRGS is seen in emission (solid line) as well as absorption (dashed line).

Evidence of mechanical feedback is also detected in optical lines from ionized gas, with shock velocities $\approx 500 \mathrm{~km} \mathrm{~s}^{-1}$ (Davis et al. 2012).

\section{OBSERVATIONS}

Our observations include Herschel Fourier Transform Spectrometer (SPIRE-FTS) spectroscopy obtained as part of the "Beyond The Peak" (BTP; OT1_jsmith1; PI: J. D. Smith) survey of 21 nuclear regions and 2 extra-nuclear regions selected from the SINGS (Kennicutt et al. 2003) and KINGFISH (Kennicutt et al. 2011) surveys. BTP provides deep, intermediate spaced mapping of a large collection of nearby galaxies at ${ }^{12} \mathrm{CO}$ transitions above $J=(3-2)$.

\subsection{PACS Spectroscopy}

NGC 1266 has been imaged in [C II], [O I], [O III], and [N $\mathrm{II}$ ] emission lines as part the Herschel key program KINGFISH (Kennicutt et al. 2011). The data were using the KINGFISH pipeline for PACS spectroscopy in HIPE v9.0.3063 using the calibration files in PACS calVersion 41, following the methods described in Croxall et al. (2012).

\subsection{SPIRE-FTS Spectroscopy}

All SPIRE-FTS observations use a four-point dither which sub-critically samples extended emission with an internal jiggle mirror. Our data are calibrated with HIPE v10 using an extended-flux calibration. The default pipeline includes a central, unjiggled telescope response function (RSRF) created from thousands of repetitions of dark observations. We calculate new jiggled RSRFs from $\simeq 100$ repetitions of jiggled darks, now available in HIPE v11. For dark subtraction, we use the unjiggled deep darks obtained during the same FTS observing run and fit a high-order polynomial to each bolometer.

While NGC 1266 is a compact source, the Long Wavelength Spectrometer (SLW) bolometer beam size and dither pattern produce repeat, but off-center SLW observations of our target. At this time, dithered calibrations have only been obtained for extended sources. To calibrate these dithered observations we begin with the Jiggle-0 (undithered) spectrum, processed with the point-source pipeline. Next all data were re-processed as with mapped calibrations, and an average spectrum was created by weighting individual SLW bolometers with the projected overlap of the beam profile and a $20^{\prime \prime}$ aperture centered on the peak of the SPIRE $250 \mu \mathrm{m}$ image (Kennicutt et al. 2011). We ratio the mapped and point-source spectra to create a transfer function to calibrate the dithered observations while simultaneously accounting for the beam filling factor of a point source. Off target bolometers also sample ample sky around NGC 1266 and we find no continuum or line emission, and thus perform no background subtraction.

The resulting spectrum (Figure 1) shows prominent lines of $\left[\mathrm{N}_{\mathrm{II}}\right],\left[\mathrm{C}_{\mathrm{I}}\right], 10$ transitions of ${ }^{12} \mathrm{CO}$, and 7 transitions of $\mathrm{H}_{2} \mathrm{O}$. We measure emission line fluxes from the unapodized spectrum using sinc profiles with a variable spectral width. Due to outflows we allow line central velocities to vary up to $100 \mathrm{~km} \mathrm{~s}^{-1}$ from the systematic velocity. The line fluxes and errors associated with the continuum uncertainty are listed in Table 1, which also includes ground based measurements of ${ }^{12} \mathrm{CO} J=(1-0),(2-1)$, and (3-2) from Alatalo et al. (2011). In addition to the $\mathrm{H}_{2}$ lines from Spitzer IRS, we also add NIR 1-0 S(1) $\mathrm{H}_{2}$ fluxes from Riffel et al. (2013).

The Table 1 line fluxes are extinction-corrected using the PAHFIT fully mixed geometry decomposition of NGC 1266's low resolution IRS spectroscopy, which yielded a silicate opacity $\tau_{9.7 \mu \mathrm{m}}=2.05$. The correction if we adopt a screen geometry is negligibly different.

\section{POWERING MOLECULAR EMISSION}

We consider energy sources that are capable of powering the emission from the central $100 \mathrm{pc}$ of NGC 1266: an AGN and SF. X-ray emission from an obscured AGN could produce an XDR, while SF would produce a PDR. Both phenomena are also capable of driving shocks, which would mechanically heat the gas. However, we emphasize that the dominance of one heating mechanism does not preclude the presence of the other.

\section{1. $\mathrm{CO}$ and $\mathrm{H}_{2}$ \\ 3.1.1. Shocks}

Motivated by shocks detected in optical lines, and molecular outflows, we first consider shocked gas as the source of $\mathrm{CO}$ excitation. To fit the CO SLED, we form combinations of the J- and/or C-shock models from Flower \& Pineau Des Forêts (2010). We then fit these to observations after normalization by total intensity from $J=(1-0)$ to (13-12) according to

$$
I_{\text {norm }}\left(J_{i}\right)=\sum_{k} f_{k}\left({ }^{12} \mathrm{CO}\right) \times \frac{I_{k}\left(J_{i}\right)}{\sum_{i=1}^{13} I_{k}\left(J_{i}\right)},
$$

where the $k$ th model contributes a fraction $f_{k}$ to the total predicted CO intensity, with a minimum $f_{k}=0.01$ and $\sum_{k} f_{k}=1$. 
Table 1

NGC 1266 IRS, PACS, and SPIRE-FTS Extinction-corrected Line Fluxes

\begin{tabular}{|c|c|c|c|c|}
\hline Species & Trans & $\begin{array}{l}\text { Wave } \\
(\mu \mathrm{m})\end{array}$ & $\begin{array}{c}\text { Flux } \\
\left(10^{-17} \mathrm{~W} \mathrm{~m}^{-2}\right)\end{array}$ & Inst. \\
\hline${ }^{12} \mathrm{CO}$ & $1-0$ & 2600 & $0.063 \pm 0.004$ & 1 \\
\hline${ }^{12} \mathrm{CO}$ & $2-1$ & 1300 & $0.39 \pm 0.015$ & 1 \\
\hline${ }^{12} \mathrm{CO}$ & $3-2$ & 867.0 & $1.02 \pm 0.11$ & 1 \\
\hline${ }^{12} \mathrm{CO}$ & $4-3$ & 650.7 & $2.69 \pm 1.14$ & 2 \\
\hline${ }^{12} \mathrm{CO}$ & $5-4$ & 520.6 & $3.84 \pm 0.69$ & 2 \\
\hline${ }^{12} \mathrm{CO}$ & $6-5$ & 433.9 & $4.28 \pm 0.35$ & 2 \\
\hline${ }^{12} \mathrm{CO}$ & $7-6$ & 371.9 & $4.76 \pm 0.28$ & 2 \\
\hline${ }^{12} \mathrm{CO}$ & $8-7$ & 325.5 & $4.71 \pm 0.36$ & 2 \\
\hline${ }^{12} \mathrm{CO}$ & $9-8$ & 289.3 & $4.64 \pm 0.65$ & 2 \\
\hline${ }^{12} \mathrm{CO}$ & $10-9$ & 260.4 & $3.94 \pm 0.60$ & 2 \\
\hline${ }^{12} \mathrm{CO}$ & $11-10$ & 236.8 & $4.03 \pm 0.61$ & 2 \\
\hline${ }^{12} \mathrm{CO}$ & $12-11$ & 217.1 & $2.85 \pm 0.30$ & 2 \\
\hline${ }^{12} \mathrm{CO}$ & $13-12$ & 200.4 & $2.65 \pm 0.58$ & 2 \\
\hline $\mathrm{H}_{2} \mathrm{O}$ & $2_{11}-2_{02}$ & 398.9 & $1.09 \pm 0.32$ & 2 \\
\hline $\mathrm{H}_{2} \mathrm{O}$ & $2_{02}-1_{11}$ & 303.7 & $2.91 \pm 1.04$ & 2 \\
\hline $\mathrm{H}_{2} \mathrm{O}$ & $3_{12}-3_{03}$ & 273.4 & $1.80 \pm 0.40$ & 2 \\
\hline $\mathrm{H}_{2} \mathrm{O}$ & $3_{12}-2_{21}$ & 260.2 & $1.83 \pm 0.61$ & 2 \\
\hline $\mathrm{H}_{2} \mathrm{O}$ & $3_{21}-3_{12}$ & 258.0 & $2.38 \pm 0.45$ & 2 \\
\hline $\mathrm{H}_{2} \mathrm{O}$ & $2_{20}-2_{11}$ & 244.1 & $1.69 \pm 0.45$ & 2 \\
\hline $\mathrm{H}_{2} \mathrm{O}$ & $4_{22}-4_{13}$ & 248.2 & $<0.47$ & 2 \\
\hline $\mathrm{H}_{2} \mathrm{O}$ & $5_{23}-5_{14}$ & 212.5 & $<0.47$ & 2 \\
\hline$\left[\mathrm{C}_{\mathrm{I}}\right]$ & $\ldots$ & 370.7 & $3.19 \pm 0.26$ & 2 \\
\hline$\left[\mathrm{C}_{\mathrm{I}}\right]$ & $\ldots$ & 609.6 & $1.13 \pm 0.86$ & 2 \\
\hline$[\mathrm{C}$ II $]$ & $\ldots$ & 157.7 & $17.30 \pm 0.46$ & 3 \\
\hline [O I] & $\ldots$ & 63.18 & $11.40 \pm 1.32$ & 3 \\
\hline$[\mathrm{N}$ II $]$ & $\ldots$ & 205 & $1.70 \pm 0.88$ & 2 \\
\hline $\mathrm{H}_{2}$ & $\mathrm{~S}(4)$ & 8.026 & $21.90 \pm 1.01$ & 4 \\
\hline $\mathrm{H}_{2}$ & $\mathrm{~S}(3)$ & 9.662 & $13.00 \pm 0.39$ & 4 \\
\hline $\mathrm{H}_{2}$ & $\mathrm{~S}(2)$ & 12.282 & $10.90 \pm 0.26$ & 4 \\
\hline $\mathrm{H}_{2}$ & $\mathrm{~S}(1)$ & 17.035 & $8.48 \pm 0.10$ & 4 \\
\hline $\mathrm{H}_{2}$ & $\mathrm{~S}(0)$ & 28.171 & $2.94 \pm 0.22$ & 4 \\
\hline $\mathrm{H}_{2}$ & $1-0 \mathrm{~S}(1)$ & 2.1213 & $10.90 \pm 0.20$ & 5 \\
\hline
\end{tabular}

Notes. Ground ${ }^{12} \mathrm{CO}$ from ${ }^{1}$ Alatalo et al. (2011). Lines observed with ${ }^{2}$ SPIREFTS, ${ }^{3}$ PACS, ${ }^{4}$ Spitzer-IRS instruments. NIR $\mathrm{H}_{2}$ from ${ }^{5}$ Riffel et al. (2013). IRS fluxes have been extracted over a $10^{\prime \prime}$ aperture centered on the peak of the IR continuum emission which is unresolved by Spitzer and Herschel.

With a large reservoir of dense gas we expect some level of SF in NGC 1266. The observed TIR emission is consistent with $\mathrm{SF} \approx 2.1 M_{\odot} \mathrm{yr}^{-1}$ (Alatalo et al. 2011). So we consider combinations of shock and plane-parallel PDR models. To estimate the emission from PDRs, we adopt the models of Wolfire et al. (2010), with updates in Hollenbach et al. (2012). Note that we adopted the shock and PDR models with solar abundance as calculated. We do not include the 60/100 $\mu \mathrm{m}$ ratio from the dust spectral energy distribution as a constraint due to uncertainties in the optical depth of the dust emission. However, we do constrain $\beta_{\mathrm{CO}}=L_{\mathrm{CO}} / L_{\mathrm{TIR}}$ not to exceed the observed ratio, based upon the assumption that shocks do not contribute appreciably to $L_{\mathrm{TIR}}$ and that a single face-on PDR contributes a continuum with an intensity related to the far-UV radiation field $G_{0}$ as

$$
I_{\mathrm{TIR}}\left(G_{0}\right) \simeq \frac{3 \times 1.6 \times 10^{-3}}{4 \pi} \times G_{0}\left(\mathrm{erg} \mathrm{s}^{-1} \mathrm{~cm}^{-2} \mathrm{sr}^{-1}\right) .
$$

A PDR fits the lowest- $J$ CO lines with $n(\mathrm{H})=10^{3.5} \mathrm{~cm}^{-3}$, $\log G_{0}=2.25$, while $\mathrm{C}$-shocks fit the higher- $J$ lines with $v=30 \mathrm{~km} \mathrm{~s}^{-1}, n(\mathrm{H})=2 \times 10^{4} \mathrm{~cm}^{-3}$ (reduced $\chi^{2}=2.6$ ). The best-fitting combination of the existing models are shown in Figure 2(a) with a solid line (black), as well as the individual PDR (blue) and shock (red) components. Model parameter probability distribution functions are shown on the right. The NGC $1266 \mathrm{H}_{2} \mathrm{O}$ SLED is also shown top-right. Although it was not used as an input, the best-fit model reproduces it well, including the faint high temperature transitions. The shock under-predicts the $\left[\mathrm{O}_{\mathrm{I}}\right]$ 63, but the $\left[\mathrm{O}_{\mathrm{I}}\right]$ intensity varies strongly with shock parameters. A small contribution from a $10 \mathrm{~km} \mathrm{~s}^{-1}$ shock we would explain the observed [O I] intensity.

\subsection{2. $P D R+P D R$}

A dual-PDR model $\left(n_{1}(\mathrm{H})=10^{3.75} \mathrm{~cm}^{-3}, \quad G_{0,1}=10^{6}\right.$; $n_{2}(\mathrm{H})=10^{5.5}, G_{0,2}=10^{3.5}$ ) could equally well explain the ${ }^{12} \mathrm{CO}$ and $\mathrm{H}_{2}$ excitation and relative intensity. We reject the dualPDR scenario because it requires $G_{0} \approx 10^{4}-10^{6}$, resulting in $\beta_{\mathrm{CO}} 1000 \times$ lower than that observed. Meijerink et al. (2013) similarly rejected a dual PDR in NGC 6240 based on this argument. In NGC 1266, a dual PDR under-predicts $\beta_{\mathrm{CO}}$, and hence over-predicts $L_{\mathrm{TIR}}$, by three orders of magnitude. With $\beta$ constrained to $\geqslant 4.6 \times 10^{-4}$, the resulting reduced $\chi^{2}$ is $\geqslant 170$. Thus a two component PDRs is rejected as the sole source of the molecular emission.

\subsection{3. $X D R$}

The ROSAT X-ray flux of $2.49 \times 10^{-13} \pm 0.3 \mathrm{erg} \mathrm{s}^{-1} \mathrm{~cm}^{-2}$ from $0.2-2.0 \mathrm{keV}$ (White et al. 1995) could also indicate an XDR contribution to ${ }^{12} \mathrm{CO}$ and the $\mathrm{H}_{2} \mathrm{O}$ lines detected in our spectrum. We will fully explore the possible contribution of XDRs in our full survey, which includes $10 \mathrm{AGNs}$, in a future paper. However, an XDR is unlikely to dominate the ${ }^{12} \mathrm{CO}$ emission in NGC 1266. First, XDRs produce high ionization fractions in molecular gas leading to $\mathrm{H}_{2} \mathrm{O}^{+}$and $\mathrm{OH}^{+}$emission with comparable intensities to $\mathrm{H}_{2} \mathrm{O}$ lines (van der Werf et al. 2010; Meijerink et al. 2013), yet no emission from these molecules is detected. We do detect absorption from $\mathrm{H}_{2} \mathrm{O}^{+}$, but this has been seen in systems where XDR are ruled out (e.g., Arp220; Rangwala et al. 2011).

Second, $\beta_{\mathrm{CO}}$ also eliminates XDRs as the dominant heating source in NGC 1266. The efficiency at which X-rays are converted to line emission and IR continuum is very different than in shocks which heat gas but are relatively inefficient at heating dust. This is illustrated in Figure 3 , a plot of $\beta_{\mathrm{CO}}$ against $\beta_{[\mathrm{CI}]}$ and $\beta_{\mathrm{H}_{2} \mathrm{O}}$. As discussed in Meijerink et al. (2013), XDR and PDR models have an upper limit on the line-to-continuum ratio of $\beta_{\mathrm{CO}} \leqslant 10^{-4}$, represented by the dashed line in Figure 3. In NGC 1266 that ratio for the 13 detected CO lines is nearly $10^{-3}$, just below that of NGC 6240 (see Figure 3). Alatalo et al. (2011) found that the observed $\beta_{\mathrm{H}_{2}}$ ratio was three times larger than could explained by an XDR. The ratio of $\beta_{[\mathrm{CI}]}$ is also enhanced by an order of magnitude in NGC 1266, as in NGC 6240. The linearity of [C I] with ${ }^{12} \mathrm{CO}$, seen in Figure 3, suggests it is a powerful diagnostic especially useful where the entire ${ }^{12} \mathrm{CO}$ is not observable, as is the case with high redshift galaxies observed with ALMA.

\subsection{Shocked $\mathrm{H}_{2} \mathrm{O}$}

The gas phase abundance of $\mathrm{H}_{2} \mathrm{O}$ is driven by two processes: formation via endothermic reactions and the release from ices on the surface of dust grains. J- and C-shocks, as well as XDRs, are capable of producing the warm and dense molecular gas characteristic of $\mathrm{H}_{2} \mathrm{O}$ emitting regions.

$\mathrm{H}_{2} \mathrm{O}$ emission is not included in the Wolfire et al. (2010) PDR models because it is very faint. Therefore, we assume 

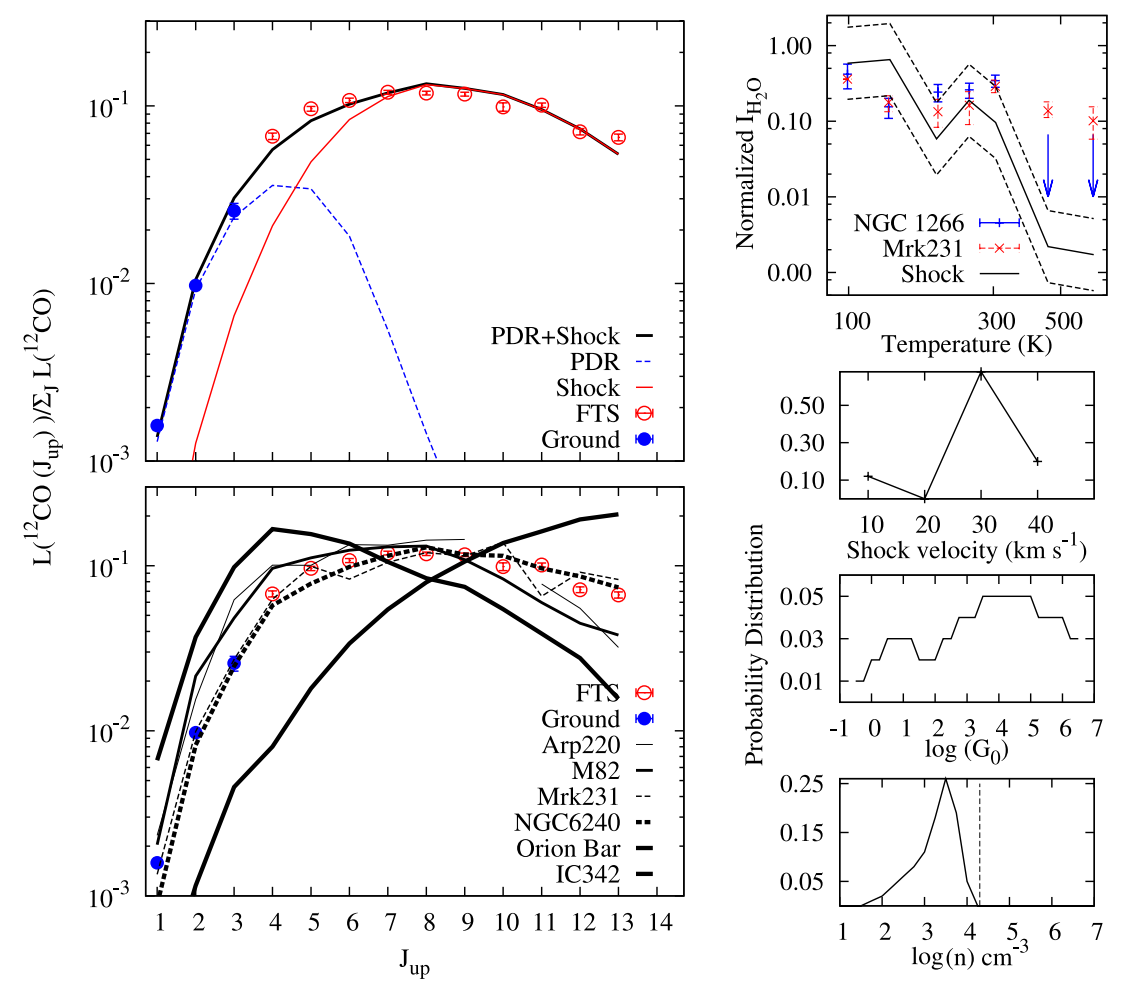

Figure 2. Top-left: NGC $1266{ }^{12} \mathrm{CO}$ SLED (up to $J=(13-12)$; circles) normalized by total observed ${ }^{12} \mathrm{CO}$ luminosity. Shown is the best-fitting PDR (dashed blue) and shock model (solid red). Bottom-left: comparison of ${ }^{12} \mathrm{CO}$ SLEDs of various galaxies, and the Orion Bar. Right: the $\mathrm{H}_{2} \mathrm{O}$ SLED includes NGC 1266 , Mrk231 and the best-fit shock model with a factor of three uncertainty in individual lines. Normalized probability distributions functions of the PDR+Shock parameters, and the $\mathrm{H}_{2} \mathrm{O}$ SLED. A vertical dashed-line represents the only pre-shock density that fits observations.

(A color version of this figure is available in the online journal.)

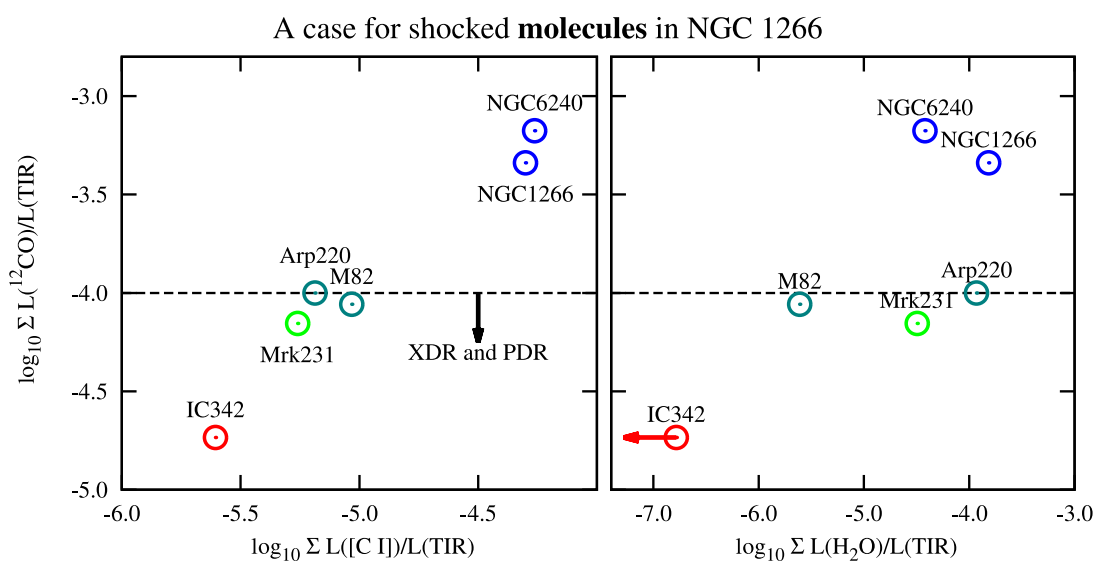

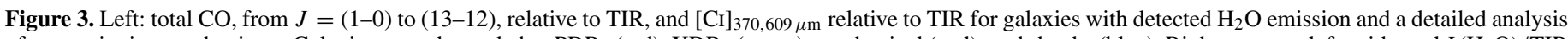
of gas excitation mechanisms. Galaxies are color coded as PDRs (red), XDRs (green), mechanical (teal), and shocks (blue). Right: same as left, with total $L\left(\mathrm{H}_{2} \mathrm{O}\right) / \mathrm{TIR}$ on the $x$-axis. The horizontal dashed line marks the upper limit of $L(C O) / L(T I R)$ for XDRs and PDRs (Meijerink \& Spaans 2005; Meijerink et al. 2007).

(A color version of this figure is available in the online journal.)

the Orion Bar value of $L\left(\mathrm{H}_{2} \mathrm{O}\right) / L(\mathrm{CO})=0.001$ (Habart et al. 2010) for our modeled PDRs. The observed parameters of the Orion Bar PDR $G_{0} \approx 4 \times 10^{4}$ (Tielens \& Hollenbach 1985) and $n(\mathrm{H}) \approx 10^{5} \mathrm{~cm}^{-3}$ (Allers et al. 2005; Pellegrini et al. 2009) are of higher density and excitation than the PDR parameters necessary to explain the low excitation CO in NGC 1266. These energetic conditions favor the formation of water, and thus provide an upper limit to the $\mathrm{H}_{2} \mathrm{O}$ emission we expect from the PDR.

Table 2 and Figure 3 summarize the ratio of $\mathrm{H}_{2} \mathrm{O},{ }^{12} \mathrm{CO}$, and [C I] to $L$ (TIR). We examine the ratio of $\mathrm{H}_{2} \mathrm{O}$ to ${ }^{12} \mathrm{CO}$ because both originate in molecular gas, unlike the IR continuum. The
Shock+PDR combination predicts a $L\left(\mathrm{H}_{2} \mathrm{O}\right) / L(\mathrm{CO})=0.34$, very similar to the NGC 1266 value of 0.33 . The observed value is also similar to that found in the XDR heated Mrk231 (van der Werf et al. 2010), and the mechanically heated Arp220 (Rangwala et al. 2011). Thus, total $L\left(\mathrm{H}_{2} \mathrm{O}\right)$ relative to $L(\mathrm{CO})$ or $L$ (TIR) is limited as a shock diagnostic because it can be enhanced by radiative pumping in systems with high gas column densities, resulting in bright emission from high levels of $\mathrm{H}_{2} \mathrm{O}$. However, in a sample of IR pumped galaxies, typical $\mathrm{H}_{2} \mathrm{O}$ transitions are $\mathrm{H}_{2} \mathrm{O} 2_{02}-1_{11} / 4_{22}-4_{13} \leqslant 2.5$ (Yang et al. 2013). In Figure $2 \mathrm{H}_{2} \mathrm{O} 4_{22}-4_{13}$ and $5_{23}-5_{14}$ are undetected in NGC 1266, with $202-1_{11} / 4_{22}-4_{13} \geqslant 4.0$. Thus, the intense emission and 
Table 2

Line to Continuum Ratios

\begin{tabular}{|c|c|c|c|c|c|}
\hline Object & $\begin{array}{l}L(\mathrm{TIR}) \\
\times 10^{10}\end{array}$ & $\begin{array}{c}L(\mathrm{CO}) / L(\mathrm{TIR}) \\
\times 10^{-5}\end{array}$ & $\begin{array}{c}L\left(\mathrm{H}_{2} \mathrm{O}\right) / L(\mathrm{TIR}) \\
\times 10^{-7}\end{array}$ & $\begin{array}{c}L([\mathrm{C} \mathrm{I}]) / L(\mathrm{TIR}) \\
\times 10^{-6}\end{array}$ & Heating \\
\hline IC $342^{\mathrm{a}}$ & 1.3 & 1.77 & 1.59 & 2.5 & PDR \\
\hline $\mathrm{M} 82^{\mathrm{b}}$ & 5.6 & 8.75 & 24.5 & 9.3 & Mech \\
\hline NGC $6240^{c}$ & 75 & 66.7 & 380 & 54.7 & Shock \\
\hline NGC $1266^{\mathrm{d}}$ & 2.4 & 45.8 & 1534 & 50.0 & Shock \\
\hline $\operatorname{Mrk} 231^{\mathrm{e}}$ & 400 & 7.00 & 321 & 5.5 & XDR \\
\hline $\operatorname{Arp} 220^{f}$ & 200 & 10.0 & 1170 & 6.5 & Mech \\
\hline
\end{tabular}

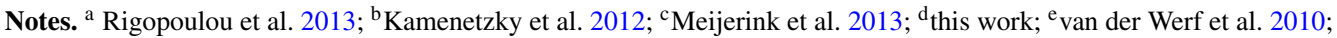

${ }^{\mathrm{f}}$ Rangwala et al. 2011.

weak excitation of $\mathrm{H}_{2} \mathrm{O}$ emission provides strong evidence for shock heating of molecular gas in NGC 1266.

\section{DISCUSSION}

\subsection{Driving Shocks: Star-formation or an AGN}

The shock model which fits the CO SLED converts $70 \%$ of incident mechanical power into observable $\mathrm{H}_{2}$ emission. Taking the observed $L\left(\mathrm{H}_{2}\right)=1.5 \times 10^{7} L_{\odot}$, this requires a incident mechanical luminosity of $2.1 \times 10^{7} L_{\odot}$.

Alatalo et al. (2011) argued for an AGN based on the ratio of mass outflow to SF rate of $\dot{M}_{\text {out }} / \dot{M}_{\mathrm{SF}}=5$. However, recent models used to explain massive outflows in high redshift galaxies find SF driven outflow rates between $1-8 \times \mathrm{SF}$ rate (Bournaud et al. 2013), suggesting SF could dominate. To determine the possible contribution from SF we assume the core SF rate is $2.1 M_{\odot} \mathrm{yr}^{-1}$ (Alatalo et al. 2011). From Hopkins et al. (2011), the energy injection rate into a turbulent medium is

$$
\dot{E}=\left(1+\eta_{p} \tau\right) \times 4 \times 10^{-4} \times \mathrm{SF} \times \mathrm{c} \delta v .
$$

We assume radiation and winds contribute equally to the momentum injection rate $\left(\eta_{p}=2\right)$, neglecting supernovae. $\tau$ is the optical depth of IR radiation, weighted by the momentum of each photon, which we conservatively take to be unity. $\delta v$ is the width of the gas velocity distribution. Adopting the core CO line widths of $100 \mathrm{~km} \mathrm{~s}^{-1}$, we find $\dot{E}(\mathrm{SF})=1.3 \times 10^{7} L_{\odot}$. Thus, given uncertainties in $\tau$ and $\eta_{p}$, a SF of $2.1 M_{\odot} \mathrm{yr}^{-1}$ could sustain the energetics of the derived shocks indefinitely.

The mechanical energy injected by jets from the central radio source has also been estimated assuming the central source is an AGN (K. Nyland et al., in preparation). The implied jet power is $2.5-14 \times 10^{8} L_{\odot}$, more than sufficient to power the shocks. Thus we find SF, an AGN, or the combination of the two, can drive the observed shocks.

\subsection{Dual PDRs}

A dual PDR is not able to simultaneously match the ${ }^{12} \mathrm{CO}$ SLED and TIR. Relaxing the constraint of $\beta_{\mathrm{CO}}$, the CO SLED can also be fit with two PDRs, representing the high density core and envelope at $100 \mathrm{pc}$, with a reduced $\chi^{2}=3.3$. This highlights that the CO SLED of NGC 1266 alone cannot unambiguously distinguish PDRs from shocks.

$\mathrm{H}_{2}$ emission also fails to distinguish a dual PDR from a $\mathrm{PDR}+$ Shock, with the total $\mathrm{H}_{2}$ intensity relative to $\mathrm{CO}$ roughly twice that observed in both models. Similarly, the individual $\mathrm{H}_{2}$ line ratios relative to total observed $\mathrm{CO}$ are similar and do not break the degeneracy between linear combinations of PDRs or shocks and PDRs.

\subsection{ULIRG Physical Conditions}

The high IR-surface-brightness and gas densities found within the core of NGC 1266 are closely analogous to the physical conditions of many ULIRGs.

Like many ULIRGs, NGC 1266 possesses an outflow in ionized and molecular gas. In ULIRGs with outflows $\mathrm{H}_{2} \mathrm{O}$ absorption lines are blueshifted by $100 \mathrm{sm} \mathrm{s}^{-1}$ relative to those in emission (i.e., Mrk231; van der Werf et al. 2010). Our data show broad [C II] emission $\left(\sigma_{v}=264 \mathrm{~km} \mathrm{~s}^{-1}\right)$, but $\mathrm{H}_{2} \mathrm{O}$ and $\mathrm{CO}$ line centers are within $40 \mathrm{~km} \mathrm{~s}^{-1}$ of each other, implying a stronger connection between $\mathrm{H}_{2} \mathrm{O}$ and ${ }^{12} \mathrm{CO}$ in NGC 1266 than in ULIRGs.

The lower than average ${ }^{13} \mathrm{CO} /{ }^{12} \mathrm{CO}$ in $\mathrm{NGC} 1266$ is also a characteristic of LIRGs and ULIRGs (Aalto et al. 1995), and indicates a low CO optical depth generally attributed to a highly turbulent medium. We speculate that in NGC 1266, a similar molecular gas velocity structure is the result of shocks. Unlike some ULIRGs, this shocked material is unlikely the result of a merger, as no extended $\mathrm{H}$ I emission has been detected (Alatalo et al. 2011). A high density is corroborated by molecular line ratios, such as $\mathrm{HCN} /{ }^{13} \mathrm{CO}$ which is an order of magnitude larger than those observed in other early-type galaxies (Crocker et al. 2012), and also similar to ULIRGs.

The infrared energy distribution observed with Spitzer and Herschel indicates the dust in NGC 1266 is heated by a radiation field with $G_{0}=20$. Assuming a spherical distribution of gas and dust with $N(\mathrm{H}) / A_{\mathrm{V}}=5.3 \times 10^{-22} \mathrm{mag} \mathrm{\textrm {cm } ^ { 2 }}$, a radius of $60 \mathrm{pc}$, and $\tau(\mathrm{Si}) / A_{\mathrm{V}}=20$ (Chiar \& Tielens 2006), we find $A_{\mathrm{V}} \sim 10^{3} \mathrm{mag}, A_{8 \mu \mathrm{m}}=38$, and $\tau(\mathrm{Si}) \approx 50$ to the center of NGC 1266. This is much larger than observed, and whichever physical process energizes the IR continuum, the observed $(\approx 10 \mathrm{~s} \mu \mathrm{m})$ continuum emission cannot originate at the center of NGC 1266 behind the highest possible column.

\section{CONCLUSIONS}

The dominant energetic source of gas excitation within NGC 1266 is challenging to determine. The extreme column densities of dust are capable of hiding otherwise unambiguous diagnostics of AGN or SF. SPIRE-FTS observations of mid$J \mathrm{CO}$ and $\mathrm{H}_{2} \mathrm{O}$ emission now provide the strongest evidence to date that the molecular gas of NGC 1266 is energized by a $\approx 30 \mathrm{~km} \mathrm{~s}^{-1} \mathrm{C}$-shock with a pre-shock density of $\approx 2 \times 10^{4} \mathrm{~cm}^{-3}$. A PDR produces the low- $J$ CO emission, amounting to 0.14 of the observed CO luminosity. This may originate from the diffuse envelope surrounding the $60 \mathrm{pc}$ core.

The discovery of shock-excited $\mathrm{H}_{2} \mathrm{O}$ sets NGC 1266 and NGC 6240 apart from other galaxies studied in detail. Such strong emission has only been detected in extreme 
environments, i.e., Arp 220 (Rangwala et al. 2011) and Mrk 231 (van der Werf et al. 2010). Unlike these other galaxies, NGC 1266 and 6240 lack emission from line transitions above $300 \mathrm{~K}$ (Figure 2, top-right), a signature of IR pumping, leaving shocks as the dominant mechanism. $\mathrm{H}_{2} \mathrm{O}$ emission has proved to be a useful diagnostic to separate PDR and shocks, and of the galaxies compared here, NGC 1266 has the highest observed $L\left(\mathrm{H}_{2} \mathrm{O}\right) / L(\mathrm{TIR})$ ratio.

The CO line-to-continuum ratio (Meijerink et al. 2013) is a robust diagnostic of shock excitation. We propose that [C I], which scales linearly with $L(\mathrm{CO})$ over $\approx 2 \mathrm{dex}$, is also a powerful shock diagnostic. Another potential diagnostic is $\mathrm{CO} J=(7-6)$; like $\left[\mathrm{C}_{\mathrm{I}}\right]$, its ratio to total $\mathrm{CO}$ is nearly constant for all the galaxies in Figure 2. The utility of these lines will be explored further in our analysis of the entire BTP sample.

We conclude that the energetics of SF and AGN can both drive the observed shocks. A SF dominated energy source would explain the lack of high ionization potential IR emission lines usually detected in AGN, but not seen in NGC 1266 (i.e., [Ne v], [O IV]; Smith et al. 2007).

Despite having an infrared luminosity more than 30 times less than that of a ULIRG, the compact molecular gas core in NGC 1266 has strikingly similar high gas surface density, including high-excitation, shocked gas and strong outflows. Thus it is possible for the bulk of a galaxy's gas reservoir to obtain the same extreme physical characteristics of a ULIRG, even within an otherwise quiescent stellar-dominated system.

We thank the referee for their valuable contributions to the work presented. Research supported by NASA/JPL RSA 1427378(E.W.P., J.D.S., and A.F.C.), NASA JPL/Caltech 1426973(M.G.W.), DAGAL network from the People Programme (Marie Curie Actions) of the EU Seventh Framework Programme FP7/2007-2013/under REA grant agreementnumber PITN-GA-2011-289313 (J.H.K).

\section{REFERENCES}

Aalto, S., Booth, R. S., Black, J. H., \& Johansson, L. E. B. 1995, A\&A, 300, 369

Alatalo, K., Blitz, L., Young, L. M., et al. 2011, ApJ, 735, 88

Allers, K. N., Jaffe, D. T., Lacy, J. H., Draine, B. T., \& Richter, M. J. 2005, ApJ, 630,368

Bothwell, M. S., Chapman, S. C., Tacconi, L., et al. 2010, MNRAS, 405, 219

Bournaud, F., Perret, V., Renaud, F., et al. 2013, ApJ, submitted (arXiv:1307.7136)

Chiar, J. E., \& Tielens, A. G. G. M. 2006, ApJ, 637, 774

Crocker, A., Krips, M., Bureau, M., et al. 2012, MNRAS, 421, 1298

Croxall, K. V., Smith, J. D., Wolfire, M. G., et al. 2012, ApJ, 747, 81

Dale, D. A., Smith, J. D. T., Schlawin, E. A., et al. 2009, ApJ, 693, 1821

Davis, T. A., Krajnović, D., McDermid, R. M., et al. 2012, MNRAS, 426, 1574

Farrah, D., Afonso, J., Efstathiou, A., et al. 2003, MNRAS, 343, 585

Flower, D. R., \& Pineau Des Forêts, G. 2010, MNRAS, 406, 1745

Fukui, Y., \& Kawamura, A. 2010, ARA\&A, 48, 547

Habart, E., Dartois, E., Abergel, A., et al. 2010, A\&A, 518, L116

Hollenbach, D., Kaufman, M. J., Neufeld, D., Wolfire, M., \& Goicoechea, J. R. 2012, ApJ, 754, 105

Hopkins, P. F., Quataert, E., \& Murray, N. 2011, MNRAS, 417, 950

Kamenetzky, J., Glenn, J., Rangwala, N., et al. 2012, ApJ, 753, 70

Kennicutt, R. C., Jr., Armus, L., Bendo, G., et al. 2003, PASP, 115, 928

Kennicutt, R. C., Calzetti, D., Aniano, G., et al. 2011, PASP, 123, 1347

Meijerink, R., Kristensen, L. E., Weiß, A., et al. 2013, ApJL, 762, L16

Meijerink, R., \& Spaans, M. 2005, A\&A, 436, 397

Meijerink, R., Spaans, M., \& Israel, F. P. 2007, A\&A, 461, 793

Pellegrini, E. W., Baldwin, J. A., Ferland, G. J., Shaw, G., \& Heathcote, S. 2009, ApJ, 693, 285

Rangwala, N., Maloney, P. R., Glenn, J., et al. 2011, ApJ, 743, 94

Riffel, R., Rodríguez-Ardila, A., Aleman, I., et al. 2013, MNRAS, 430, 2002

Rigopoulou, D., Hurley, P. D., Swinyard, B. M., et al. 2013, MNRAS, 434, 2051

Smith, J. D. T., Draine, B. T., Dale, D. A., et al. 2007, ApJ, 656, 770

Tielens, A. G. G. M., \& Hollenbach, D. 1985, ApJ, 291, 747

van der Werf, P. P., Isaak, K. G., Meijerink, R., et al. 2010, A\&A, 518, L42

White, N. E., Gimmi, N. E., \& Angelini, L. 1995, The WGACAT Version of the ROSAT PSPC Catalogue, Rev. 1 (1wga), http://heawww.gsfc. nasa.gov/users/white/wgacat/wgacat.html CDS

Wolfire, M. G., Hollenbach, D., \& McKee, C. F. 2010, ApJ, 716, 1191

Yang, C., Gao, Y., Omont, A., et al. 2013, ApJL, 771, L24 